\title{
Person-Centered Care for Older Adults living in Long-term care facilities: A Systematic Literature Review
}

\author{
Jasmine Yee ${ }^{1}$, Marina Celly Martins Ribeiro Souza ${ }^{2 *}$, Natália de Cássia Horta ${ }^{3}$ \& Constance Kartoz ${ }^{4}$ \\ ${ }^{1}$ Undergraduate Student, Department of Public Health, The College of New Jersey, United States. \\ ${ }^{2 *}$ Associate Professor, Department of Public Health, The College of New Jersey, United States. \\ ${ }^{3}$ Associate Professor, Department of Medicine. Pontifical Catholic University of Minas gerais, Brazil. \\ ${ }^{4}$ Associate Professor. Department of Nursing, The College of New Jersey, United States.
}

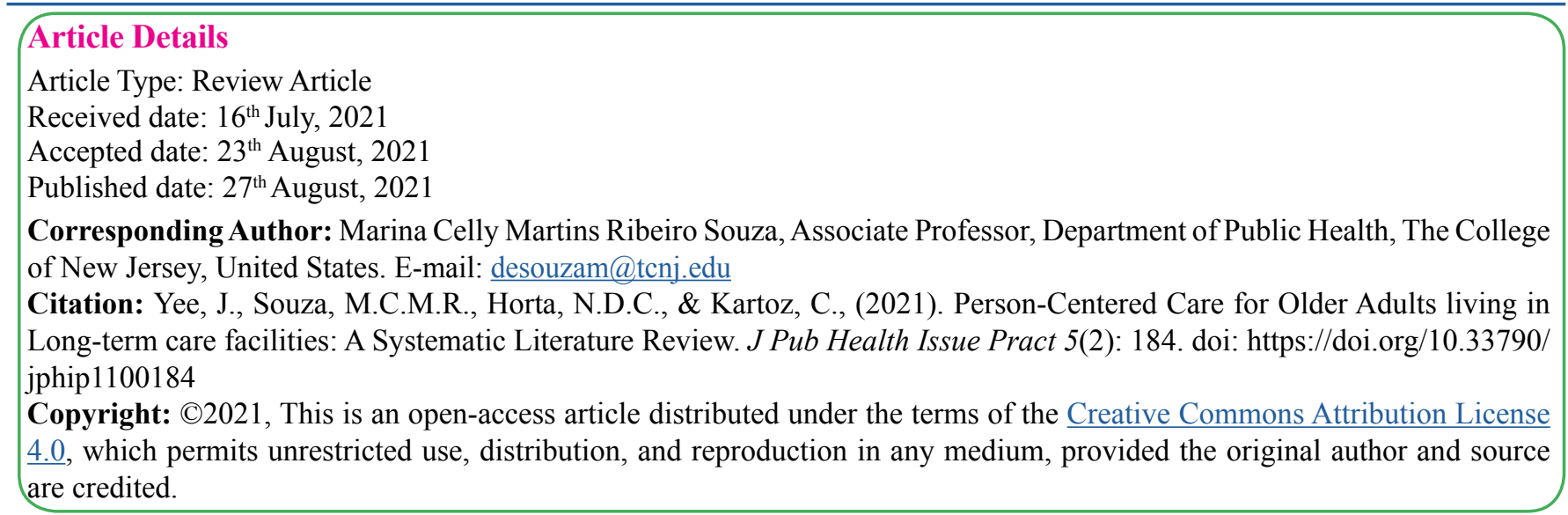

\section{Abstract}

Person-centered care (PCC) is the empowering approach of holistic care that shifts from a traditional biomedical framework to one that emphasizes older adults' personalized preferences, abilities, and strengths. This systematic literature review aimed to describe the current status of research on PCC for older adults living in longterm care facilities (LTCFs). The method that conducted the search involved using 5 consistent keywords along with adding various descriptor terms to help narrow the search. A total of 18 articles were discussed in the final review after meeting all of the inclusion criteria. The results showed overall beneficial outcomes of PCC for institutionalized older adults, the importance of prioritizing residents' preferences when implementing PCC, and perspectives of residents and staff workers on the state of PCC in their respective LTCFs, as well as what they think are barriers and facilitators. Identifying what the top shared preferences are is the first step to providing individualized PCC delivery that will improve the quality of care and quality of life for older adults living in LTCFs. Furthermore, taking into account both staff and residents' perspectives will lead to an improved PCC climate in LTCFs that will enable better care outcomes.

Key words: Person-centered care, patient-centered care, older adults, quality of life, long-term care facilities, nursing homes.

\section{Introduction}

As countries become more developed and undergo a demographic transition, the decreasing birth rates, higher life expectancies, amongst other factors, result in population aging. Consequently, the number of older adults is expected to grow at an increasing rate. This change in population structure leads to a heightened need for long-term care facilities (LTCFs) that can provide quality housing and care for older adults [1]. An approach that is quickly gaining momentum among healthcare providers is person-centered care (PCC), which is a type of model that shifts from a traditional biomedical framework focusing on treating disease and illness to one that relies on understanding the various perspectives of LTCF residents while incorporating their individualized preferences, experiences, beliefs, values, and needs into their care [2]. The PCC model emphasizes empowering residents to be more involved with their own care, and staff workers are urged to adapt and respond accordingly to the great diversity of resident preferences [3]. Fostering harmonizing relationships between residents and staff workers through communication and respect in addition to providing a homelike environment in the institutions are but a few essential components to the implementation of PCC for older adults living in LTCFs [4].

Although significant positive associations of PCC with higher quality of life have been found for patients with chronic conditions and cognitive impairments such as dementia $[5,6]$ and Alzheimer's disease [7], more research is needed to examine the implementation of PCC on older adults living in LTCFs. This includes a more in-depth exploration of residents' quality of life outcomes, satisfaction with care, and following residents' preferences in order to improve PCC in LTCFs. Furthermore, due to the complexity of PCC interventions and their wide range of outcomes, there is a need for standardized, valid and reliable instruments that can measure PCC in LTCFs from the perspective of residents, family, and staff [8]. There is also a gap in publications on how PCC can impact LTCF workers, for their job satisfaction and well-being can in turn affect their provision of quality PCC for residents, directly influencing residents' quality of life and satisfaction with care [9]. Thus, the perspectives of both LTCF residents and staff workers should be examined more.

In this sense, the objective of this systematic literature review is to describe the current status of research on PCC for older adults living in LTCFs. By finding out what is already known about PCC for institutionalized older adults, this review will contribute to stakeholders to better implement and improve PCC climates in LTCFs, enabling greater care outcomes.

\section{Materials and Methods}

This systematic literature review includes qualitative and quantitative studies as well as other literature reviews to provide guidance toward understanding PCC for older adults living in LTCFs. Through the use of articles, studies, and reports, multiple definitions and overarching themes of PCC among institutionalized older adults were found. Relevant literature was discovered through searches made in scientific databases including: Pubmed, Cochrane Library, and Medline. These databases were searched utilizing the key words (("Person-centered care" OR "Patient-centered care") AND (Older Adults OR Elderly)) AND (Long-Term Care Facilities) AND (X). Following these key 
terms, descriptor words were also included at the end (in place of X). Descriptor words consisted of (quality of life, well-being, wellness, care quality, quality of care, service quality, care outcomes, patient outcomes, patient health, [Beliefs, opinions, attitudes, perspectives], care delivery, care satisfaction, satisfaction with care, effectiveness, effects, impacts, literature review, qualitative studies, quantitative studies, holistic care). "Nursing homes" was used as an additional keyword with "Long-Term Care Facilities", while "Institutionalized Older Adults/Residents" were also used in addition to "Older Adults" OR "Elderly".

The initial literature search retrieved 2,163 potential papers. After the first phase of paper selection based on titles that suggested using the concept of PCC and older adults living in a LTCF, 555 papers remained. Removal of duplicate papers resulted in 101 relevant titles being selected. The next phase of paper selection consisted of reading the abstracts for relation to the subject, which narrowed these titles down to 34 . Of these abstracts, 18 of the full text papers met the inclusion criteria and were ultimately included in this systematic literature review. The inclusion criteria consisted of 1) having an electronic abstract and full text available, 2) focusing on the specific target population and setting (older adults living in LTCFs), and 3) using the actual concept of PCC (i.e. not just using the term). Papers with preliminary results and failure to fully address the topic were excluded. Languages such as English, Spanish, and Portuguese were incorporated to the search for relevant literature, as 2 authors are fluent in these languages. Articles found in Cochrane were already found in Pubmed. Medline gave no relevant search results. A summary of this search is represented on Figure 1.

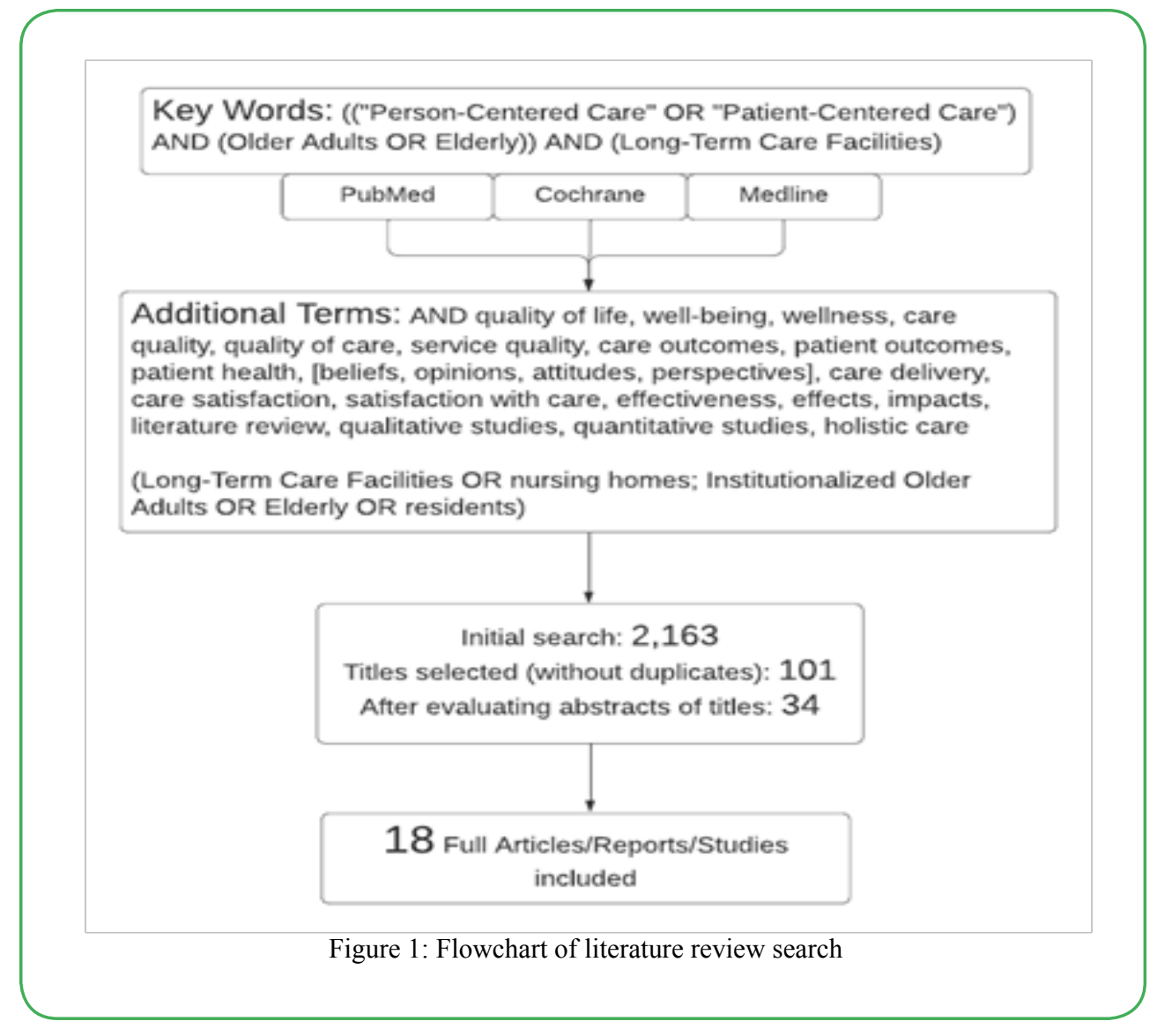

\section{Results and Discussion}

The final selection of 18 relevant papers detailing PCC for institutionalized older adults is included in this systematic literature review. The articles ranged from 2007 to 2020 . After reading all of the studies, they were grouped thematically into 3 categories. The largest group of studies explored the perspectives of staff and residents $(n=$ 10) with most of these being qualitative in nature. Another category involved studies of outcomes $(\mathrm{n}=5)$ with the majority here being quantitative in nature. Finally, a small selection of papers $(n=3)$ detailed preferences of the residents and staff in terms of PCC. Moreover, differing perspectives of residents and staff workers were explored to give insight on how well PCC has been implemented in LTCFs worldwide and what they think the barriers and facilitators are to PCC.

\section{Person-centered Care Outcomes in Long-term Care Facilities}

Overall, PCC has been found to have beneficial impacts for older adults living in LTCFs as indicated by quality of life/care satisfaction scores, as well as cognitive and psychological functioning. In a Swedish national survey filled out by both residents and staff workers,
PCC had a significant positive association with residents' quality of life as quality of life rated among residents increased [1]. These results were further corroborated by Edvardsson et al. [5] who found that residents who participated in everyday activities (such as making coffee, setting or clearing the table, watering plants, and participating in outdoor walks) on their own lived in more person- centered units and had significantly higher quality of life and higher cognitive scores than residents who did not participate in everyday activities. Additional findings also suggested an improved psychological status of older adults, as person-centered interventions were associated with lower rates of boredom and feelings of helplessness [9]. In a longitudinal, retrospective cohort study for nursing homes in Kansas, residents' satisfaction with overall quality of life and quality of care were higher in homes that had fully implemented PCC [10], while in another survey for Midwestern U.S. nursing home residents, a significant positive correlation with PCC environment and residents' life satisfaction was also concluded [11].

Another area of interest is the effect that PCC has on the LTCF staff workers themselves, which appear to be similarly beneficial. For instance, a significant negative association between PCC and 
perceived job strain was found among staff [1]. Brownie \& Nancarrow [9] conceptualized that PCC may reduce job strain through personal satisfaction and the ability to deliver individualized care. These findings should prompt further studies on the interaction between PCC, staff satisfaction, quality outcome measures, and patient satisfaction.

In light of this, it seems that PCC benefits both residents and staff. The study conducted by Sköldunger et al. [1] has shown that working in a PCC manner supports and enhances residents' autonomy with regard to mobility, self-care, and daily activities, all of which contributed to a higher quality of life for the residents.

\section{Residents' Care Preferences}

A large component of PCC in LTCFs involves taking into account the preferences that residents have when receiving care [3]. Bangerter et al. [3] analyzed residents' care preferences and found they ranged from interpersonal interactions with staff, coping strategies, personal care, and healthcare discussions. Most notably, LTCF residents seemed to prioritize choosing who they can involve in their own care and having favorable interactions with the staff workers. For example, when receiving mental health services, residents often prefer to talk to their family, friends, and nursing home staff rather than a mental health professional [12]. In addition, staff workers showing respect and demonstrating that they truly care about the wellbeing of the residents were key to residents perceiving a positive relationship with the staff [3].

Similarly, Abbott et al. [13] found in their assessment interviews with nursing home residents and older adults receiving home and community-based services that more than $90 \%$ of the respondents in each group rated "having regular contact with family" as a significant priority. Majority of the respondents also agreed that having privacy, choices about what to eat, when to bathe, and activity options were important preferences for them as well [13].

However, fulfilling residents' preferences such as selecting their medical professional or choosing who they can involve in their own care were usually restricted by barriers in the facility itself [12]. As a result, Bangerter et al [12] suggested that LTCFs incorporate policies enabling residents' choices and increasing resident autonomy. These policies should allow for more family involvement and integration in residents' lives, and more options should be provided for residents in terms of healthcare providers and everyday care. Furthermore, conducting evaluations of residents' satisfaction with quality of care and services can aid in the assurance of meeting residents' preferences [3].

Understanding residents' care preferences is crucial to providing and improving PCC for institutionalized older adults. Overall, findings indicate that honoring residents' preferences is essential if LTCFs are to achieve the goal of providing PCC for older adults. Assessing and adapting to the diverse care preferences of older adults can serve as a guide when delivering PCC in LTCFs, but this can only be done when the perceptions of residents are known, acknowledged, and considered.

\section{Residents and Staff Perspectives on Person-centered Care}

Investigating how key healthcare stakeholders perceive the current state of PCC in LTCFs is vital to improving this individualized, holistic care strategy for facilities seeking to incorporate it. Specifically, focusing on the perspectives of residents and staff workers can play a significant role in providing PCC within a particular LTCF. Understanding and comparing these two stakeholders perspectives can also provide insight as to why certain activities restrict PCC in LTCFs. Alternatively, these articles discuss methods to better facilitate and enhance the PCC care model for institutionalized older adults.

Through a qualitative examination on how residents perceive and adapt to the care they receive in LTCFs claiming to provide PCC,
Donnelly \& MacEntee [14] found that negative comments dominated, especially about how residents felt that the institution and its policies restricted their freedom. Namely, bathing and toilet policies prevented residents from bathing whenever and how often they desired, time constraints tended to neglect their emotional needs, lack of privacy challenged maintenance of personal dignities, and staff imposition of activities or care led to losses of autonomy [14]. Although sympathetic to their working load, residents still felt distant from the staff. As a result, residents generally challenged claims of PCC in their LTCFs by citing reasons of lack of empathy, human indignities, and violations of personal autonomy [14].

Akin to these findings, another qualitative study with 31 nursing home stakeholders (residents, family members, advanced practice registered nurses, physicians, and administrators) in a current patientcentered medical care, revealed that economic and profit-motivated constraints placed by the LTCF system limited medical resident involvement opportunities. Conflicting goals among different stakeholders for patient-centered medical care rendered PCC difficult to deliver [15].

To further highlight differences in perspectives, a cross-sectional study that collected data in 2016 from both nursing home residents and staff in China determined that residents generally perceived the PCC climate of their respective nursing homes at a lower level than the staff, and residents in larger nursing homes perceived a better climate of PCC than those in smaller nursing homes [16]. Particularly, the results revealed that residents sensed a relatively low level of being welcomed in the care home, while staff strongly indicated that the home was a caring community for residents [16].

Additionally, questionnaires measuring person-centeredness were developed by Zimmerman et al. [17] and split between residents and staff. For residents, four factors were included in their questionnaire: well-being and belonging, individualized care and services, social connectedness, and atmosphere. For staff, five factors were included: workforce practices, social connectedness, individualized care and services, atmosphere, and caregiver-resident relationships. With all the factors combined in each questionnaire, they found that the staff rated a higher overall mean score of person-centeredness than the residents did in their questionnaires, thus reflecting how residents perceived the implementation and effects of PCC to a lesser degree than the staff [17].

Other studies found that older adults had a more positive, yet wary attitude toward purported PCC in their LTCFs. For example, residents of a LTCF in Ireland considered their PCC climate setting to be hospitable, welcoming, clean and safe. However, they acknowledged that psychosocial concerns about adapting to living in LTCFs needed to be addressed, particularly among younger male residents [18]. Furthermore, in a case report by Harrison \& Frampton [19] during which they observed residents' perspectives of PCC in 10 U.S. nursing homes that adopted PCC as a model of care, residents attributed the increased choice in wake and bedtimes, being heard by organizational leaders, and consistent staff assignment as positive changes since PCC was incorporated. However, the residents still perceived inconsistent PCC aspects resembling institutional life in their nursing homes, such as long response times, infrequent access to nature, and a lack of transparency around illness and death [19].

Overall, the discrepancy between residents and staff opinions regarding the status of PCC in LTCFs shows that each group has different interests and values on what makes an ideal PCC environment. Moreover, the lower mean scores for PCC from residents indicate the need for new policies, investigations, and communications geared towards their preferences [16]. When different stakeholder groups have conflicting perceptions of PCC climates and prioritize different facets of person-centeredness, the delivery of PCC can be made difficult. Because of this, stakeholders should have a shared understanding and consensus for what PCC should be like in a LTCF.

JPHIP, an open access journal ISSN- 2581-7264 


\section{Limitations}

This literature review contains several limitations. Due to the novelty of PCC, there is a paucity of papers available. Specifically, the lack of research of PCC for older adults living in LTCFs complicates efforts for conducting a full analysis of this strategy in this particular population and setting. Another limitation of this review comes from excluding databases that could have been used for this research. Since the majority of the articles were extracted from PubMed, potential papers for this review may have been missed.

\section{Conclusions}

Identifying what the top shared preferences are is the first step to providing individualized PCC delivery that will improve quality of care and quality of life for older adults living in LTCFs. Furthermore, taking into account both staff and residents' perspectives will lead to an improved PCC climate in LTCFs that will enable better care outcomes. Additionally, PCC seems to alleviate care burden among staff, presenting itself as a worthy investment that maintains the wellbeing of LTCF residents and staff. More studies should be done on PCC outcome measures, its cost-effectiveness, whether PCC can be applied globally in various cultural lenses, and staff turnover in PCC facilities over time. Finally, the tools and challenges for measuring and evaluating PCC for older adults in LTCFs need more research, as PCC should be treated as a care strategy that leads to specific and measurable outcomes. Although PCC has been garnering more attention, it appears that the voices of residents are still ignored in many cases, with older adults settling to cope with an institutionalized environment.

Conflict of interests: The author declares no conflict of interest.

\section{References}

1. Sköldunger, A., Sandman, P. O., \& Backman, A. (2020). Exploring person-centred care in relation to resource utilization, resident quality of life and staff job strain - findings from the SWENIS study. BMC geriatrics, 20(1), 465. https://doi. org/10.1186/s12877-020-01855-7

2. Jones C. S. (2011). Person-centered care. The heart of culture change. Journal of gerontological nursing, 37(6), 18-25. https://doi.org/10.3928/00989134-20110302-04

3. Bangerter, L. R., Van Haitsma, K., Heid, A. R., \& Abbott, K. (2016b). "Make Me Feel at Ease and at Home": Differential Care Preferences of Nursing Home Residents. The Gerontologist, 56(4), 702-713. https://doi.org/10.1093/geront/gnv026

4. Koren M. J. (2010). Person-centered care for nursing home residents: the culture-change movement. Health affairs (Project Hope), 29(2), 312-317. https://doi.org/10.1377/ hlthaff.2009.0966

5. Edvardsson, D., Petersson, L., Sjogren, K., Lindkvist, M., \& Sandman, P. O. (2014). Everyday activities for people with dementia in residential aged care: associations with personcentredness and quality of life. International journal of older peoplenursing,9(4),269-276.https://doi.org/10.1111/opn.12030

6. Sjögren, K., Lindkvist, M., Sandman, P. O., Zingmark, K., \& Edvardsson, D. (2013). Person-centredness and its association with resident well-being in dementia care units. Journal of advanced nursing, 69(10), 2196-2205. https://doi.org/10.1111/ jan. 12085

7. Edvardsson, D., Winblad, B., \& Sandman, P. O. (2008). Personcentred care of people with severe Alzheimer's disease: current status and ways forward. The Lancet. Neurology, 7(4), 362-367. https://doi.org/10.1016/S1474-4422(08)70063-2

8. Crandall, L. G., White, D. L., Schuldheis, S., \& Talerico, K. A. (2007). Initiating person-centered care practices in long-term care facilities. Journal of gerontological nursing, 33(11), 47 56. https://doi.org/10.3928/00989134-20071101-08

J Pub Health Issue Pract

Volume 5. 2021. 184
9. Brownie, S., \& Nancarrow, S. (2013). Effects of person-centered care on residents and staff in aged-care facilities: a systematic review. Clinical interventions in aging, 8, 1-10. https://doi. org/10.2147/CIA.S38589

10. Poey, J. L., Hermer, L., Cornelison, L., Kaup, M. L., Drake, P., Stone, R. I., \& Doll, G. (2017). Does Person-Centered Care Improve Residents' Satisfaction With Nursing Home Quality? Journal of the American Medical Directors Association, 18(11), 974-979. https://doi.org/10.1016/j.jamda.2017.06.007

11. Yoon J. Y. (2018). Relationships among person-centered care, nursing home adjustment, and life satisfaction: a cross-sectional survey study. International psychogeriatrics, 30(10), 15191530. https://doi.org/10.1017/S1041610218000194

12. Bangerter, L. R., Abbott, K., Heid, A. R., Klumpp, R. E., \& Van Haitsma, K. (2016a). Health Care Preferences Among Nursing Home Residents: Perceived Barriers and Situational Dependencies to Person-Centered Care. Journal of gerontological nursing, 42(2), 11-16. https://doi. org/10.3928/00989134-20151218-02

13. Abbott, K. M., Klumpp, R., Leser, K. A., Straker, J. K., Gannod, G. C., \& Van Haitsma, K. (2018). Delivering PersonCentered Care: Important Preferences for Recipients of Long-term Services and Supports. Journal of the American Medical Directors Association, 19(2), 169-173. https://doi. org/10.1016/j.jamda.2017.10.005

14. Donnelly, L., \& MacEntee, M. I. (2016). Care Perceptions among Residents of LTC Facilities Purporting to Offer PersonCentred Care. Canadian journal on aging $=$ La revue canadienne du vieillissement, 35(2), 149-160. https://doi.org/10.1017/ S0714980816000167

15. Garcia, T. J., Harrison, T. C., \& Goodwin, J. S. (2016). Nursing Home Stakeholder Views of Resident Involvement in Medical Care Decisions. Qualitative health research, 26(5), 712-728. https://doi.org/10.1177/1049732315573206

16. Yang, Y., Li, H., Xiao, L. D., Zhang, W., Xia, M., \& Feng, H. (2019). Resident and staff perspectives of person-centered climate in nursing homes: a cross-sectional study. $B M C$ geriatrics, 19(1), 292. https://doi.org/10.1186/s12877-0191313-x

17. Zimmerman, S., Allen, J., Cohen, L. W., Pinkowitz, J., Reed, D., Coffey, W. O., Reed, P., Lepore, M., Sloane, P. D., \& University of North Carolina-Center for Excellence in Assisted Living Collaborative (2015). A measure of person-centered practices in assisted living: the PC-PAL. Journal of the American Medical Directors Association, 16(2), 132-137. https://doi. org/10.1016/j.jamda.2014.07.016

18. Kelly, F., Reidy, M., Denieffe, S., \& Madden, C. (2019). Older adults' views on their person-centred care needs in a longterm care setting in Ireland. British journal of nursing (Mark Allen Publishing), 28(9), 552-557. https://doi.org/10.12968/ bjon.2019.28.9.552

19. Harrison, J., \& Frampton, S. (2017). Resident-Centered Care in 10 U.S. Nursing Homes: Residents' Perspectives. Journal of nursing scholarship : an official publication of Sigma Theta Tau International Honor Society of Nursing, 49(1), 6-14. https://doi. org/10.1111/jnu.12247 